\title{
Hypertension and blood pressure variability management practices among physicians in Singapore
}

This article was published in the following Dove Press journal:

Vascular Health and Risk Management

17 July 2017

Number of times this article has been viewed

\author{
Sajita Setia' \\ Kannan Subramaniam² \\ Jam Chin Tay ${ }^{3}$ \\ Boon Wee Teo ${ }^{4}$ \\ 'Chief Medical Office, Medical Affairs, \\ Pfizer Pte Ltd, Singapore; ${ }^{2}$ Global \\ Medical Affairs, Asia Pacific Region, \\ Pfizer Australia, West Ryde, NSW, \\ Australia; ${ }^{3}$ Department of General \\ Medicine, Tan Tock Seng Hospital, \\ ${ }^{4}$ Department of Medicine, Yong Loo \\ Lin School of Medicine, National \\ University of Singapore, Singapore
}

Correspondence: Sajita Setia Medical Affairs, Pfizer Pte Ltd, 80 Pasir Panjang Road, \#16-8I/82, Mapletree Business City, Singapore 117372

Tel +6564038754

Fax +6567224188

Email sajita.setia@pfizer.com
Purpose: There are limited data on blood pressure variability (BPV) in Singapore. The absence of updated local guidelines might contribute to variations in diagnosis, treatment and control of hypertension and BPV between physicians. This study evaluated BPV awareness, hypertension management and associated training needs in physicians from Singapore.

Materials and methods: Physicians from Singapore were surveyed between September 8, 2016 , and October 5, 2016. Those included were in public or private practice for $\geq 3$ years, cared directly for patients $\geq 70 \%$ of the time and treated $\geq 30$ patients for hypertension each month. The questionnaire covered 6 main categories: general blood pressure (BP) management, BPV awareness/diagnosis, home BP monitoring (HBPM), ambulatory BP monitoring (ABPM), BPV management and associated training needs.

Results: Responses from 60 physicians (30 general practitioners [GPs], 20 cardiologists, 10 nephrologists) were analyzed (77\% male, 85\% aged 31-60 years, mean 22 years of practice). Approximately $63 \%$ of physicians considered white-coat hypertension as part of BPV. The most common diagnostic tool was HBPM (overall 77\%, GPs 63\%, cardiologists $65 \%$, nephrologists $70 \%$ ), but ABPM was rated as the tool most valued by physicians ( $80 \%$ overall), especially specialists (97\%). Withdrawn Singapore guidelines were still being used by $73 \%$ of GPs. Approximately $48 \%$ of physicians surveyed did not adhere to the BP cutoff recommended by most guidelines for diagnosing hypertension using HBPM (>135/85 mmHg). Hypertension treatment practices also varied from available guideline recommendations, although physicians did tend to use a lower BP target for patients with diabetes or kidney disease. There were a number of challenges to estimating BPV, the most common of which was patient refusal of ABPM/HBPM. The majority of physicians ( $82 \%$ ) had no training on BPV, but stated that this would be useful. Conclusion: There appear to be gaps in knowledge and guideline adherence relating to the assessment and management of BPV among physicians in Singapore.

Keywords: hypertension, blood pressure, guidelines, antihypertensives, blood pressure variability, blood pressure monitoring

\section{Introduction}

Hypertension is an important risk factor for stroke and coronary heart disease and is a significant public health issue. ${ }^{1,2}$ At least $45 \%$ of deaths due to heart disease and $51 \%$ of deaths due to stroke have been attributed to hypertension. ${ }^{2}$ The global prevalence of hypertension in 2008 in adults aged 25 and over was approximately 40\%, and elevated blood pressure (BP) is estimated to cause 7.5 million deaths each year worldwide. ${ }^{1}$ Hypertension is also highly prevalent in Singapore, where it affects about 1 in 4 residents aged 30-69 years and half of those aged 60-69 years. ${ }^{3}$ 
Effective treatment of hypertension has been shown to reduce the risk of myocardial infarction by $15-25 \%$, stroke by $35-40 \%$ and heart failure by as much as $64 \% .{ }^{4-6}$ All the current international guidelines recommend 4 main drug classes for treating hypertension: angiotensin-converting enzyme inhibitors (ACEIs), angiotensin receptor blockers (ARB), calcium channel blockers (CCB) and diuretics (Table 1)..$^{7-10}$

However, not all documents are consistent in their recommended target BP values for different patient groups due to a lack of consensus from clinical trial data. This is particularly the case for patients with comorbidities such as diabetes or chronic kidney disease (CKD), in whom BP control is particularly important (Table 1).

It was previously recommended that the target BP for patients with CKD or diabetes mellitus was $\leq 130 / 80 \mathrm{mmHg} .{ }^{4}$ However, the Eighth Joint National Committee (JNC8) guidelines from the USA now suggest a more conservative target of $<140 / 90 \mathrm{mmHg}$, which is the same as the recommendation for general population patients aged $<60$ years (Table 1 ). ${ }^{7}$ In the European Society of Hypertension/European Society of
Cardiology (ESH/ESC) guidelines, the diastolic BP target is $5 \mathrm{mmHg}$ lower $(<140 / 85 \mathrm{mmHg}),{ }^{8}$ taking into account the results of the Hypertension Optimal Treatment (HOT) study and the UK Prospective Diabetes Study (UKPDS) trials (Table 1). ${ }^{11-14}$ Japanese guidelines also include lower BP targets for diabetes and CKD patients, ${ }^{10}$ mostly likely taking the high risk of stroke in Japan into account. ${ }^{15}$

It is widely accepted that lowering BP to at least below the guideline-mandated targets is required to realize the benefits of antihypertensive therapy, particularly in patients with comorbidities such as diabetes or CKD. ${ }^{16,17}$ However, despite better understanding of the causes and consequences of hypertension and the availability of a range of effective antihypertensive agents, optimal BP control is often not achieved, even in those with important comorbidities. ${ }^{18-21}$ For example, nearly two-thirds $(64.5 \%)$ of treated elderly patients with hypertension in Singapore had suboptimal BP control, ${ }^{22}$ and only $36.6 \%$ of older patients and $62.1 \%$ of those with stable CKD achieved a systolic BP (SBP) target of $<140 \mathrm{mmHg}^{21}$

Measurement of mean BP alone may not provide a complete picture of cardiovascular risk in an individual.

Table I Recommendation from international guidelines commonly referred to in Singapore

\begin{tabular}{|c|c|c|c|c|}
\hline & NICE $20 \mathrm{I}^{9}$ & ESH/ESC $2013^{8}$ & JNC8 $2014^{7}$ & JSH $2014^{10}$ \\
\hline Definition of hypertension & $\geq 140 / 90$ "and" daytime & $\geq 140 / 90$ & ND & $\geq 140 / 90$ (clinic) \\
\hline \multirow[t]{2}{*}{$(\mathrm{mmHg})$} & ABPM or HBPM average & & & $\geq 135 / 85$ (HBPM) \\
\hline & $\mathrm{BP} \geq 135 / 85$ & & & $\geq 130 / 80$ (ABPM) \\
\hline \multirow{2}{*}{\multicolumn{5}{|c|}{$\begin{array}{l}\text { Initiation of drug therapy } \\
(\mathrm{mmHg})\end{array}$}} \\
\hline & & & & \\
\hline \multirow[t]{2}{*}{ Overall } & $\geq 160 / 100$ or daytime & $\geq 140 / 90$ & $\geq 140 / 90($ age $<60 y)$ & $\geq 140 / 90$ \\
\hline & $A B P M \geq 150 / 95$ & & $\geq 150 / 90($ age $\geq 60 y)$ & \\
\hline DM pts & ND & $\mathrm{SBP} \geq 140$ & $\geq 140 / 90$ & $\geq 130 / 80$ \\
\hline CKD pts & ND & $\mathrm{SBP} \geq 140$ & $\geq 140 / 90$ & $\geq 130 / 80$ \\
\hline \multirow[t]{6}{*}{ Recommended agents } & ACEI or ARB (age $\leq 55$ y) & Diuretics, ACEI, BB, CCB & Thiazide-type diuretics, & \\
\hline & CCB (age $>55$ y) & and $\mathrm{ARBs}$ as mono- or & $C C B, A C E I$ or $A R B$ for initial & \\
\hline & & combination therapy & treatment (including DM pts); & \\
\hline & & ACEI, ARB (DM pts) & include $A C E I$ or $A R B$ for CKD & \\
\hline & & ACEI, ARB (renal & pts & \\
\hline & & dysfunction pts) & & \\
\hline Preferred diuretic & Thiazide-type agents* & Thiazide-type agents* & Thiazide-type agents & Thiazide-like agents \\
\hline Initiate therapy with >I & ND & Pts at high risk or with & $>160 / 100$, or if SBP/DBP is & $\geq 160 / 100$ \\
\hline agent & & markedly elevated BP & $>20 />10$ above goal & \\
\hline \multirow[t]{4}{*}{ BP targets $(\mathrm{mmHg})$} & $<140 / 90$ (overall) & $<140 / 90$ & $<140 / 90($ age $<60$ y) & $<140 / 90$ (overall)** \\
\hline & $<135 / 85$ (age $<80 y)$ or & & $<150 / 90($ age $\geq 60 y)$ & $<150 / 90 \mathrm{mmHg}$ (age \\
\hline & $<145 / 85$ (age $\geq 80 y)$ using & & & $>75 y$, or $<140 / 90$, if \\
\hline & ABPM or HBPM & & & tolerated) $* *$ \\
\hline BP targets in DM (mmHg) & ND & $<140 / 85$ & $<140 / 90$ & $<130 / 80 * *$ \\
\hline BP targets in CKD (mmHg) & ND & SBP $<140(<130$ for $p t s$ & $<140 / 90$ & $<130 / 80 * *$ \\
\hline & & with overt proteinuria) & & \\
\hline Endorse significance of BPV & Yes & Yes & No & Yes \\
\hline
\end{tabular}

Notes: *Usually chlorthalidone or indapamide. **Based on clinic BP. All targets are $5 \mathrm{mmHg}$ lower for HBPM values.

Abbreviations: ABPM, ambulatory BP monitoring; ACEl, angiotensin-converting enzyme inhibitor; ARB, angiotensin receptor blocker; BB, beta-blocker; $\mathrm{BP}$, blood pressure; BPV, BP variability; CCB, calcium channel blocker; CKD, chronic kidney disease; DBP, diastolic BP; DM, diabetes mellitus; ESC, European Society of Cardiology; ESH, European Society of Hypertension; HBPM, home BP monitoring; JNC8, Eighth Joint National Committee; JSH, Japanese Society of Hypertension; ND, no data; pts, patients; NICE, National Institute for Health and Care Excellence; SBP, systolic BP; y, years. 
Oscillations in BP over both the short- and long-term, known as BP variability (BPV), were first recognized as a potential independent risk factor for adverse cardiovascular events more than 2 decades ago. ${ }^{23,24}$ Since then, numerous studies have confirmed that long-term BPV is associated with adverse cardiovascular outcomes and increased mortality, as summarized in a recent systematic review. ${ }^{25}$ The long-term BPV has also been shown to be an independent predictor of renal function deterioration in patients with hypertension, ${ }^{26}$ and visit-to-visit BPV strongly predicted all-cause, cardiovascular and non-cardiovascular mortality in patients with type 2 diabetes mellitus, independent of mean BP. ${ }^{27}$ Higher BPV has been documented in women, older patients and those with CKD or cardiovascular disease. ${ }^{28}$ There are very limited data on BPV in Singapore. A 2001 study reported that the prevalence of white-coat hypertension in young adults was higher than that of hypertension ( $2.0 \%$ vs $1.6 \%$, respectively). ${ }^{29}$ Furthermore, clinical practice related to hypertension and BPV diagnosis, treatment and control may vary substantially between physicians in Singapore given the current unavailability of local guidelines. Understanding these differences is important to inform collaborative development of local strategies to better control hypertension and BPV.

The aim of this study was to evaluate BPV awareness, clinical practice relating to hypertension management and associated training needs among physicians from Singapore.

\section{Materials and methods}

This cross-sectional survey was conducted in a random sample of physicians from Singapore over the period September 8, 2016, to October 5, 2016. Surveys were conducted according to the globally accepted standards of good clinical practice (as defined in the International Conference on Harmonization [ICH] E6 Guidelines for Good Clinical Practice, May 1, 1996), in agreement with the latest version of the Declaration of Helsinki and according to the local internal and external regulations. General practitioners (GPs), cardiologists and nephrologists were recruited using random sampling with focus on including a mix of physicians from different parts of Singapore (North, Central, North East, East and West).

\section{Study sample}

Physicians were contacted by telephone and were asked a series of questions from a short screening questionnaire to determine eligibility and willingness to participate in the survey (Supplementary material). To be eligible, physicians had to be in public (restructured) or private practice, to have been practicing for $\geq 3$ years, be involved directly in patient care $\geq 70 \%$ of the time, to personally treat $\geq 30$ patients for hypertension per month and provide verbal informed consent to participate. The physician survey was outside the scope of human biomedical research, as defined by the Bioethics Advisory Committee, Ministry of Health $(\mathrm{MOH})$, Singapore. ${ }^{30}$ The survey was carried out after approval from Pfizer's committee managing Customer Engagement Programs (CEPs). These programs are managed by a dedicated team that ensures that all adverse events (AEs) from CEPs are being reported properly and on a timely basis to safety for processing. The organization carrying out the survey (Kantar Health) was trained in AE reporting.

\section{Survey}

Quantitative surveys were conducted using a combination of online (computer-assisted web interviews [CAWI]) and face-to-face (computer-assisted personal interviews [CAPI]) interviews, each of which took approximately 30 minutes to administer. The CAWI was a self-administered interview, and CAPI was administered by a specialized health care interviewer. Two interviewers conducted all interviews after receiving identical training on the questionnaire and process.

The survey started with a brief introduction to the overall objectives of the research, followed by a series of questions covering 6 main categories (Supplementary material):

1. General BP management: 8 questions relating to hypertension guidelines in practice, target BP for different patient groups, proportions of patients achieving target BP and commonly prescribed antihypertensive agents.

2. BPV awareness and diagnosis: 3 questions relating to the definition of BPV and the diagnosis of BPV (e.g., cutoff points, techniques, and tools).

3. Home BP monitoring (HBPM): 12 questions relating to how and why physicians recommend HBPM.

4. Ambulatory BP monitoring (ABPM): 5 questions relating to how and why physicians recommend ABPM.

5. BPV management: 3 questions relating to current practice around estimating BPV, what physicians recommend and the gaps/unmet needs in this area.

6. Training needs of health care professionals regarding BPV: 9 questions relating to the extent of any existing training, the need for further education/training on BPV and what type/frequency of training would be suggested.

Interviewers recorded responses to all questions from each physician, and then these were collated and tabulated for analysis. A full copy of the study questionnaire is provided in the Supplementary material. 
This paper focuses on findings from sections 1,2 and 5 of the questionnaire related to hypertension and BPV management, and relevant parts of section 6 . Findings relating to out-of-office BP measurement practice are the subject of a separate manuscript.

\section{Statistical analysis}

Physician responses to the questionnaires were summarized using descriptive statistics (as numbers and percentages). Raw data in SPSS and dimensions (tables object module) were used for tabulation; SPSS raw data were converted into dimension format viz. mdd/ddf format. The processed tables were then checked against frequencies taken from original raw data received. Categorical variables were expressed as percentages, and continuous variables were expressed as mean values. Statistical analysis was performed using the SPSS Statistics 20 (IBM Corporation, Armonk, NY, USA).

\section{Results}

\section{Sample and response rate}

Overall, 150 GPs, 45 cardiologists and 20 nephrologists were contacted about the survey. Of those, 80, 17 and 7, respectively, did not respond when contacted for recruitment; 30, 8 and 3, respectively, declined to participate and 10,0 and 0 , respectively, saw $<30$ patients with hypertension per month. Therefore, 60 participants (30 GPs, 20 cardiologists and 10 nephrologists) were included in the final study sample (14 female [23\%], 46 male [77\%]; age: $31-40$ years [ $n=14 ; 23 \%], 41-50$ years $[n=24$; $40 \%$ ], 51-60 years $[n=13 ; 22 \%], 61-70$ years $[n=6 ; 10 \%]$ or $>70$ years $[n=3 ; 5 \%]$; mean duration of post-residence practice: 22 years; practice location: Central $[\mathrm{n}=30 ; 50 \%]$, North-East and East $[n=13 ; 22 \%]$, North $[n=12 ; 20 \%]$ or West $[n=5 ; 8 \%])$. Physician and practice characteristics and patient load by physician specialty are provided in Table 2. Cardiologists saw the highest number of patients with hypertension per month, and cardiologists and nephrologists saw more hypertensive patients with comorbidities than GPs (Table 2).

\section{BPV awareness and diagnosis}

Almost two-thirds of physicians considered white-coat hypertension and morning BP as part of BPV (Figure 1). Inclusion of white-coat hypertension in BPV was most common for GPs (83\%) and less common among specialists (45\% of cardiologist and $40 \%$ of nephrologists), whereas specialists were much more likely to include nocturnal dipping in the BPV definition ( $60 \%$ of cardiologists and $80 \%$ of nephrologists vs $33 \%$ of GPs). Morning hypertension was considered important by cardiologists $(60 \%)$ and GPs $(60 \%)$ but not by nephrologists (10\%).

Table 2 Physician and practice characteristics by specialty

\begin{tabular}{|c|c|c|c|}
\hline Characteristics & GPs $(n=30)$ & Cardiologists $(n=20)$ & Nephrologists $(n=10)$ \\
\hline Male, n (\%) & $25(83)$ & $16(80)$ & $5(50)$ \\
\hline \multicolumn{4}{|l|}{ Age range, $\mathrm{n}(\%)$} \\
\hline $31-40$ years & $4(13)$ & $3(15)$ & $7(70)$ \\
\hline $4 I-50$ years & II (37) & II (55) & $2(20)$ \\
\hline $5 I-60$ years & $9(30)$ & $3(15)$ & $I(10)$ \\
\hline $61-70$ years & $5(17)$ & $\mathrm{I}(5)$ & 0 \\
\hline$>70$ years & I (3) & $2(10)$ & 0 \\
\hline Post-residency practice duration, years & 25 & 22 & 12 \\
\hline \multicolumn{4}{|l|}{ Practice setting, n (\%) } \\
\hline GP clinic & $15(50)$ & 0 & 0 \\
\hline Group practice & $15(50)$ & 0 & 0 \\
\hline Restructured & 0 & $8(40)$ & $9(90)$ \\
\hline Private & 0 & $12(60)$ & $\mathrm{I}(10)$ \\
\hline Patients with hypertension treated each month, $n$ & 138 & 214 & 140 \\
\hline \multicolumn{4}{|l|}{ Treated patients by comorbidity* (\%) } \\
\hline No comorbidities & 37 & 17 & 16 \\
\hline Diabetes & 33 & 31 & 53 \\
\hline Hyperlipidemia & 39 & 42 & 56 \\
\hline Stroke & 6 & 13 & 13 \\
\hline Angina & 4 & 31 & 17 \\
\hline Atrial fibrillation & 3 & 18 & 12 \\
\hline Heart failure & 4 & 17 & 19 \\
\hline Myocardial infarction & 5 & 24 & 18 \\
\hline Others & 0 & 1 & 32 \\
\hline
\end{tabular}

Notes: *Total percentages may be greater than $100 \%$ as patients may have more than I comorbidity.

Abbreviation: GPs, general practitioners. 


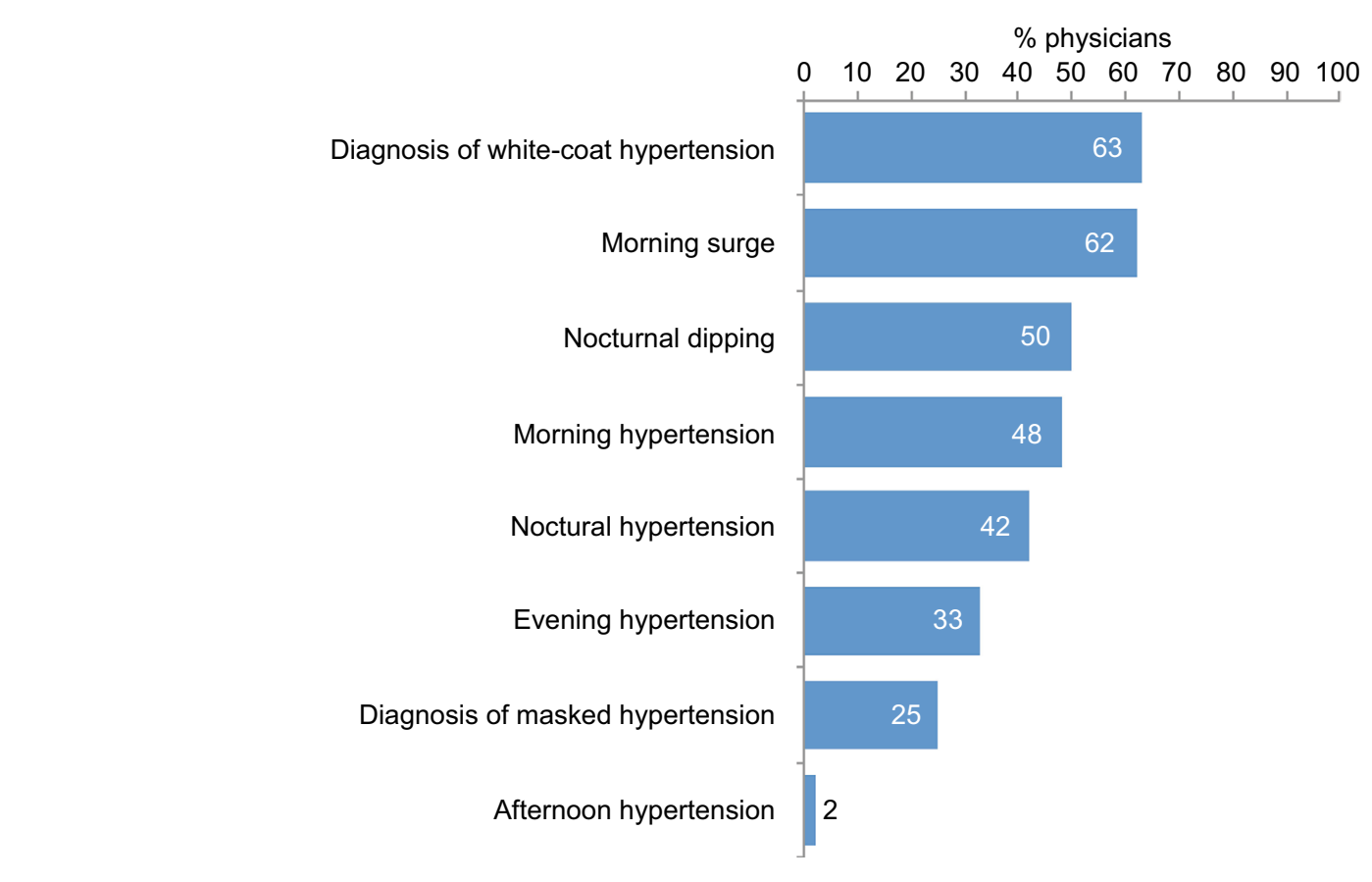

Figure I Proportion of physicians considering different factors as part of BPV. Abbreviation: BPV, blood pressure variability.

\section{HBPM and ABPM}

HBPM was the most commonly used diagnostic tool across specialties ( $77 \%$ overall; $63 \%$ of GPs, $65 \%$ of cardiologists and $70 \%$ of nephrologists). In contrast, when asked about the value placed on the different approaches, ABPM was the diagnostic tool most valued by physicians, particularly specialists ( $80 \%$ overall; $63 \%$ of GPs, $100 \%$ of cardiologists and $90 \%$ of nephrologists). Clinical BP was used (valued) less: 40\% (42\%) overall, 57\% (53\%) for GPs, 25\% (25\%) for cardiologists and $29 \%$ (40\%) for nephrologists.

\section{Hypertension diagnosis}

Almost half of all physicians surveyed did not adhere to the BP cutoff recommended by most frequently used international guidelines for diagnosing hypertension using HBPM $(>135 / 85 \mathrm{mmHg}$ ), instead using the higher clinic BP cutoff of $>140 / 90 \mathrm{mmHg}$ (Figure 2). Specialists were more likely to use the $>135 / 85 \mathrm{mmHg}$ cutoff when diagnosing hypertension using ABPM, although the proportion using the most common guideline-mandated value was still quite low $(\leq 30 \%)$ and the higher cutoff value was still used by more than one-third of GPs and cardiologists (Figure 2).

\section{Hypertension management}

Treatment approaches used by physicians varied by specialty and patient group. Initiation of antihypertensive monotherapy was most common in younger patients (age 40-60 years), reported by $74 \%$ of GPs, $62 \%$ of cardiologists and $52 \%$ of nephrologists ( $66 \%$ overall). For older patients, rates of monotherapy initiation were $52 \%, 44 \%$ and $32 \%$, respectively ( $46 \%$ overall). For patients with diabetes or kidney disease, cardiologists were more likely to initiate treatment with combination therapy ( $61 \%$ and $68 \%$, respectively), while combination therapy was most favored by nephrologists in these comorbidity patients ( $78 \%$ and $81 \%$, respectively).

For single-agent antihypertensive therapy, choice of agent depended largely on patient characteristics, but there were also some differences between specialties (Figure 3 ). Diuretics were most commonly used in older patients while those with diabetes or kidney disease most often got an ACEI or ARB (Figure 3). An ARB plus a CCB was the most commonly used drug combination across all patient groups; $58 \%$ of younger patients, $49 \%$ of older patients, $45 \%$ of those with diabetes mellitus and $41 \%$ of kidney disease patients received this combination therapy. In terms of managing BPV, the most common strategies were administration of antihypertensive medication more than once a day ( $67 \%$ of physicians) or use of combination antihypertensives (65\%).

Reported targets for initiating antihypertensive drug therapy varied by patient group, with the higher value of $>140 / 90 \mathrm{mmHg}$ used by the majority of physicians for 
Proportion of physicians using cutoff value (\%)

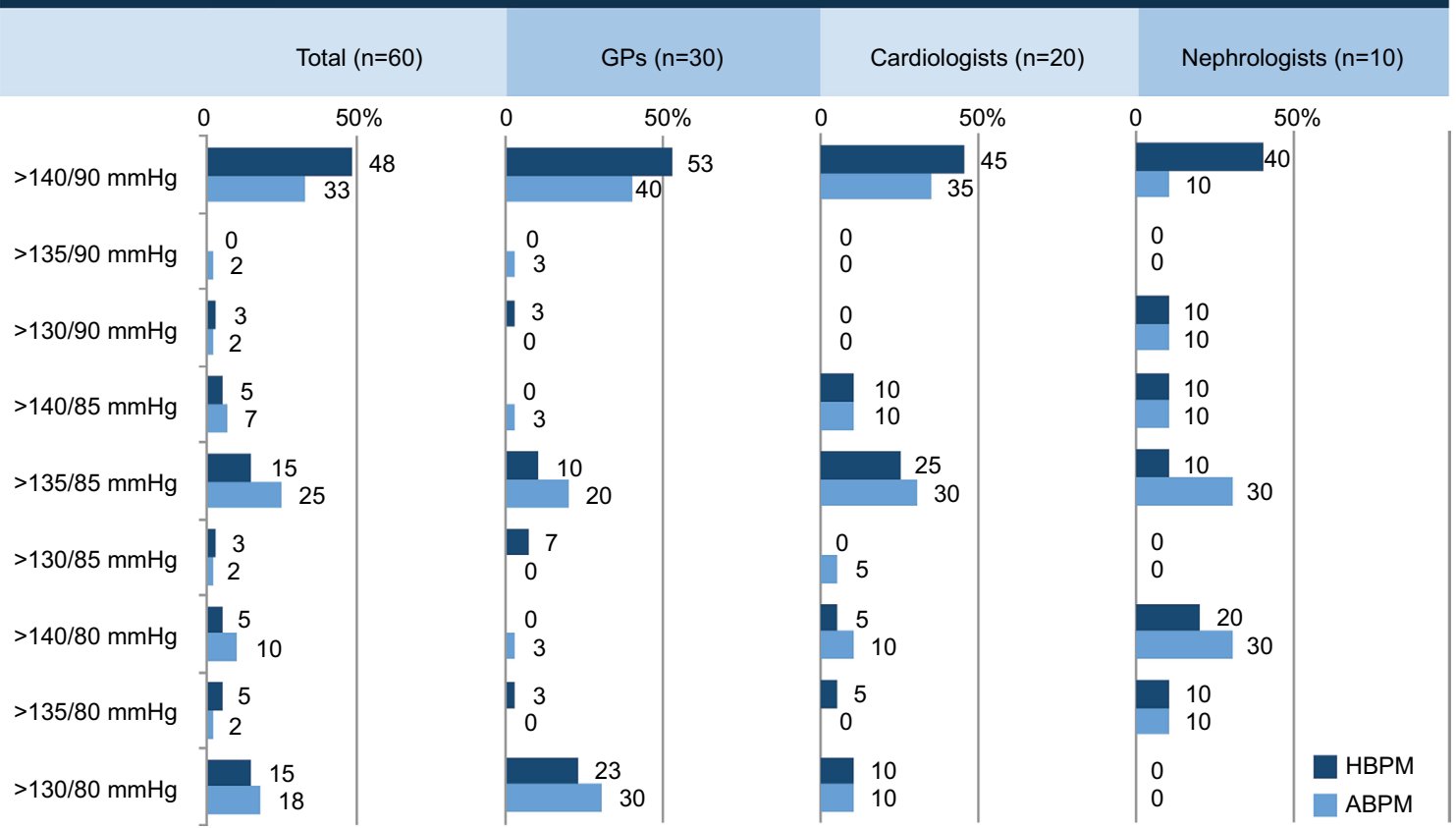

Figure 2 Proportion of physicians (overall and by specialty) using different BP cutoff values to diagnose hypertension using HBPM and ABPM (a cutoff of $>$ I35/85 mmHg is the one recommended in most guidelines).

Abbreviations: ABPM, ambulatory BP monitoring; BP, blood pressure; GPs, general practitioners; HBPM, home BP monitoring.

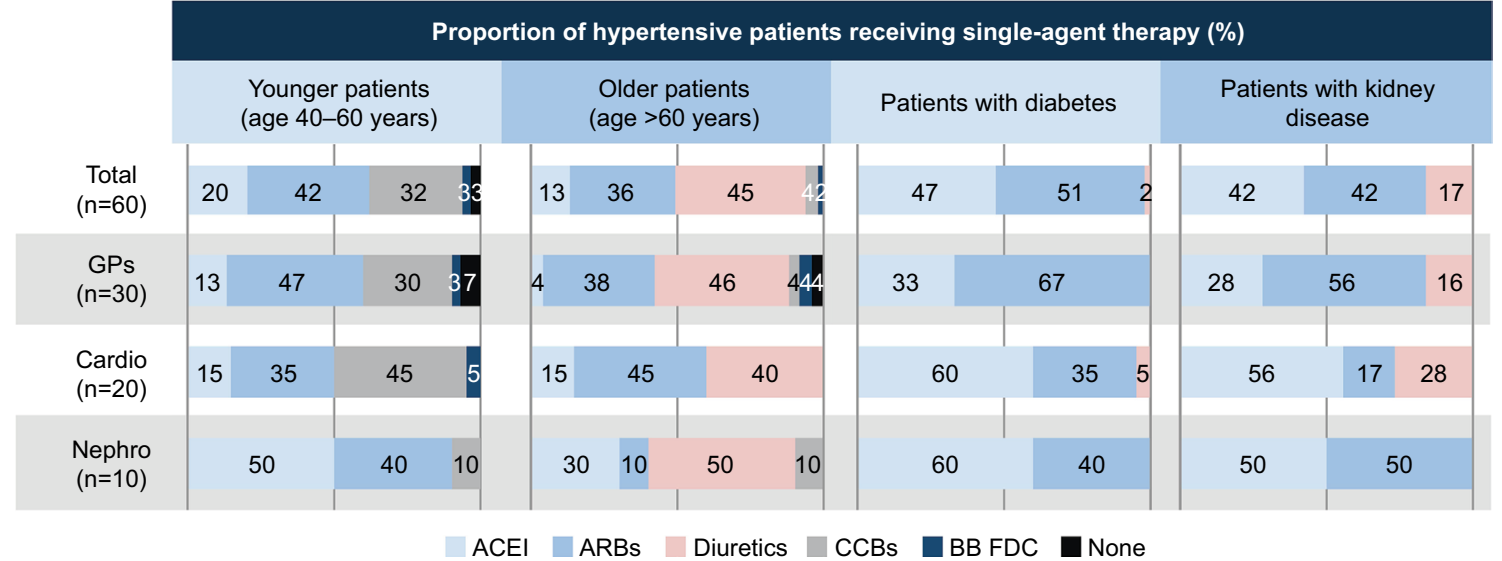

Figure 3 Drug use by patients managed using antihypertensive monotherapy.

Abbreviations: ACEI, angiotensin-converting enzyme inhibitor; ARBs, angiotensin receptor blockers; BB FDC, beta-blockers fixed dose combination; Cardio, cardiologists; CCBs, calcium channel blockers; GPs, general practitioners; Nephro, nephrologists.

patients aged 40-60 and $>60$ years, while a lower target $(>130 / 80 \mathrm{mmHg})$ tended to be used more often for patients with diabetes and, in particular, kidney disease (Figure 4).

The proportion of treated patients reported to achieve target BP differed between specialties and patient groups. For patients aged $40-60$ years, rates were $81 \%$ overall, $83 \%$ for GPs, $83 \%$ for cardiologists and 69\% for nephrologists. Corresponding values for patients aged $>60$ years were $70 \%$, $73 \%, 72 \%$ and $58 \%$, and for patients with diabetes were $72 \%$,
$74 \%, 76 \%$ and $56 \%$. Kidney disease patients had the lowest rates of target BP achievement (66\% overall, $68 \%$ for GPs and cardiologists and 57\% for nephrologists). For all patient subgroups, those seen by a nephrologist were less likely to achieve target BP compared with other specialties.

The JNC8 guidelines were most likely to be used by physicians in our survey, but nearly three-quarters of GPs still reported using the Singapore $\mathrm{MOH}$ guidelines, which are not current and have been withdrawn (Table 3 ). 
Proportion of physicians using cutoff value for antihypertensive therapy initiation (\%)

\begin{tabular}{l}
$r \begin{array}{c}\text { Younger patients } \\
\text { (age 40-60 years) }\end{array}$ \\
\hline
\end{tabular}

Figure 4 Target cutoff SBP/diastolic BP values for the initiation of drug therapy in patients with hypertension. Abbreviations: BP, blood pressure; SBP, systolic BP.

Table 3 Reported physician use of different hypertension guidelines

\begin{tabular}{lllll}
\hline Number of physicians (\%) & Total $(\mathbf{n}=\mathbf{6 0})$ & GPs $(\mathbf{n}=\mathbf{3 0})$ & Cardiologists $(\mathbf{n}=\mathbf{2 0})$ & Nephrologists $(\mathbf{n}=\mathbf{1 0})$ \\
\hline JNC8 & $36(60)$ & $13(43)$ & $16(80)$ & $7(70)$ \\
Singapore MOH $^{32 *}$ & $32(53)$ & $22(73)$ & $7(35)$ & $3(30)$ \\
ESC/ESH $^{8}$ & $27(45)$ & $9(30)$ & $15(75)$ & $3(30)$ \\
NICE $^{9}$ & $17(28)$ & $6(20)$ & $3(15)$ & $8(80)$ \\
JSH $^{10}$ & $6(10)$ & $0(0)$ & $0(0)$ & $1(10)$ \\
None & $2(3)$ & $1(3)$ & $0(0)$ & $0(0)$ \\
\hline
\end{tabular}

Notes: *Guidelines last revised in 2005 have been withdrawn and a new, updated edition is awaited.

Abbreviations: ESC, European Society of Cardiology; ESH, European Society of Hypertension; GPs, general practitioners; JNC8, Eighth Joint National Committee; JSH, Japanese Society of Hypertension; MOH, Ministry of Health; NICE, National Institute for Health and Care Excellence.

\section{Challenges and barriers}

The top 4 challenges for estimating BPV cited by physicians were patient refusal of ABPM (48\%) or HBPM (47\%), challenges in diagnosis $(43 \%)$ and lack of compliance (43\%). Affordability (35\%) and lack of resources (e.g., equipment; $33 \%$ ) were fifth and sixth on the list, while other factors (including lack of guideline recommendation, lack of time for follow-up, lack of knowledge on BPV and lack of internal support for BPV) were each cited by $<30 \%$ of physicians. When asked about solutions to these barriers, provision of subsidies to support patient access to ABPM or HBPM was the most common answer ( $63 \%$ and $60 \%$ of physicians, respectively). Approximately half of all physicians felt that patient education on HBPM and BPV and provision of resources to facilitate the diagnosis of BPV would also be helpful.

\section{Training needs}

The majority of physicians ( $\mathrm{n}=49 ; 82 \%)$ stated that they had not attended any training courses on BPV; $27 \%$ of GPs, $5 \%$ of cardiologists and $20 \%$ of nephrologists had attended training on BPV. When asked if they would like to attend training courses on BPV regularly, $87 \%$ of GPs, $55 \%$ of cardiologists and $100 \%$ of nephrologists responded affirmatively. The preferred course frequency was annual, and most said that their preferred training format was expert lectures, although cardiologists and nephrologists also expressed interest in case discussions. In terms of the topics that should be covered in training, the most popular across all specialties was "diagnosis and evaluation of BPV," followed by "medical treatment of BPV" and "interpretation of ABPM;" nephrologists expressed a specific interest in "treatment for patients with complication/comorbidity."

\section{Discussion}

Based on the results of this survey, physicians in Singapore reported a wide variety of preferences, beliefs and practices for the management of hypertension and BPV. The current absence of updated local guidelines resulted in physicians referring to several different international guidelines, but 
the proportion using common recommendations was low. Physicians also identified important training needs around BPV, especially its diagnosis and evaluation.

Our finding that a low proportion of physicians followed hypertension treatment guidelines is in line with previous data. When looking at the adherence of US-based family medicine physicians to the Seventh Joint National Committee (JNC7) guidelines for managing patients with uncontrolled $\mathrm{BP}$, the reported overall adherence rate was $53.5 \%$, with no significant improvement over time. ${ }^{31}$ Almost half of all physicians in our survey used the higher clinic BP cutoff (>140/90 $\mathrm{mmHg}$ ) to diagnose hypertension based on HBPM, for which a lower cutoff is recommended $(>135 / 85 \mathrm{mmHg}) .^{9,10}$ Both the National Institute for Health and Care Excellence (NICE) ${ }^{9}$ and Japanese Society of Hypertension $(\mathrm{JSH})^{10}$ guidelines specify the lower value for diagnosis based on HBPM, but these guidelines were the main source of information for only a minority of physicians in our survey, with more clinicians using the $\mathrm{JNC}^{7}$ and $\mathrm{ESC} /$ $\mathrm{ESH}^{8}$ guidelines, and also the withdrawn Singapore $\mathrm{MOH}$ guidelines. ${ }^{32}$ Use of the withdrawn local guidelines was highest among GPs (73\%) and lower among cardiologists (35\%) and nephrologists $(30 \%)$. The reasons behind this difference are unknown, but it may reflect greater exposure to international guidelines for specialists. Another potential contributing factor to differences between GPs and specialists could be that GPs have to deal with a greater variety of diseases than specialists. As a result, GPs may lack in-depth knowledge of hypertension compared with specialists who have an expert therapeutic focus.

In terms of drug therapy, monotherapy was most often used for younger patients in our survey. Initiation of antihypertensive therapy with a single agent is consistent with guideline recommendations, with first-line combination therapy reserved for those with markedly elevated BP (Table 1). Physicians from Singapore often used diuretic monotherapy in elderly patients and ARB monotherapy in young patients, although these choices are not specifically endorsed by any guidelines. Starting antihypertensive therapy with a combination regimen was relatively common in our survey, particularly among cardiologists and nephrologists. However, this may reflect the fact that specialists are more likely to treat patients with comorbid diseases or those who have already failed on antihypertensive monotherapy. The survey questions related to hypertension in general and did not differentiate between a new diagnosis of hypertension by GPs or specialists and management of hypertension by specialists after GP referral. Treatment approaches may be different in these 2 settings, and the inability to categorize our results based on this differentiation is a limitation of the study.

The survey responses do concur with available guidelines concerning lower BP targets for patients with CKD. However, physicians reported that these lower targets were not achieved in one-third of those with kidney disease treated with antihypertensives. Interestingly, nephrologists noted that BP control was less likely in CKD patients. Recent Singapore data from stable CKD patients also suggested that reaching BP targets is difficult. ${ }^{21}$

While the importance of treating hypertension is universally accepted, the optimal SBP target continues to be a topic of heated debate. The Systolic Blood Pressure Intervention Trial (SPRINT) reported a significantly lower risk of cardiovascular disease outcomes by targeting SBP $<120 \mathrm{mmHg}$ compared with $<140 \mathrm{mmHg}$ in patients with hypertension and high cardiovascular risk. ${ }^{33}$ However, the SPRINT excluded some subpopulations of hypertensive patients, including those with diabetes or prior stroke, aged $<50$ years and at lower cardiovascular risk. ${ }^{33}$ SPRINT findings support a lower SBP target than currently recommended for the elderly. However, data from the study may indicate the possibility of permanent renal injury due to intensive BP-lowering treatment because participants without CKD at baseline who were in the intensive group had a significantly higher rate of incident CKD ( $\geq 30 \%$ reduction in estimated glomerular filtration rate) compared with the standard treatment group. Furthermore, SPRINT excluded patients with diabetes and the results of the Action to Control Cardiovascular Risk in Diabetes Blood Pressure (ACCORD BP) trial, ${ }^{34}$ which used the same SBP target as SPRINT in a diabetic population, were confounded due to intensive glycemic control. ${ }^{35}$ In addition, there may be an intermediate SBP target at which the protective effect is greater than at the lowest extreme (which may avoid a potential J-curve phenomenon). However, this cannot be determined from the 2-group design used in SPRINT. The ongoing Optimal Blood Pressure and Cholesterol Targets for Preventing Recurrent Stroke in Hypertensives (ESH-CHLSHOT) trial $^{36}$ is exploring the protective effect of 3 different SBP treatment targets in patients with a history of stroke, including those with diabetes and will hopefully provide more insight into optimal SBP targets during antihypertensive therapy. Finally, caution should be exercised when extrapolating BP values obtained using unattended automated measurements in SPRINT to those obtained in usual clinical practice, which may be higher by up to $10 \mathrm{mmHg} .^{37,38}$

It has been suggested that effective management of BPV could contribute to both "precision medicine" (a treatment 
approach that takes individual patient genes, environment and lifestyle into account) and "anticipation medicine" (defined as an approach to disease treatment and prevention that predicts future risk by taking individual changes in markers over time and disease status in relation to behavioral and environmental factors). ${ }^{39}$ Both the ESH/ESC and JSH guidelines specifically recognize the significance of $\mathrm{BPV} .{ }^{8,10}$ Morning hypertension, evening hypertension, nocturnal hypertension, nocturnal dipping and the morning surge are types of BPV phenotypes. ${ }^{10,39}$ Most physicians in our survey regarded only morning BP surge and diagnosis of white-coat hypertension as part of BPV, although more nephrologists regarded nocturnal dipping as BPV compared with other specialties. Asians show greater morning BP surges than Westerners and the curve associating $\mathrm{BP}$ and cardiovascular event risk is steeper. ${ }^{40}$ Controlling morning BP is the first important step in achieving "perfect 24-hour BP control" in treated patients with hypertension. ${ }^{39}$ This is because the risk of cardiovascular events is highest in the morning, coinciding with trough levels of BP-lowering medication. ${ }^{39}$ It appears that the control of BPV is the most important factor, regardless of how this is achieved.

The biggest challenge in determining and monitoring BPV for Singapore-based physicians was patient refusal of ABPM or HBPM (almost half of all patients for both approaches). Better understanding may help physicians explain BPV and its assessment to patients, and to appropriately support patients through the ABPM or HBPM process. In a US-based survey, physician knowledge about the diagnosis and management of hypertension based on JNC7 guidelines was poor (only $51.2 \%$ had average baseline knowledge of 6 key concepts). ${ }^{41}$ However, completion of an online training module based on the guidelines significantly improved knowledge across all topics, ${ }^{41}$ suggesting that training can improve knowledge. These issues were reflected in our data showing that a majority of physicians would attend regular training on BPV, and almost half believed that patient education on HBPM and BPV would be useful. Affordability of BPV assessment was seen as another barrier to use of ABPM and HBPM by approximately half of the physicians in our survey, who also stated that subsidies for the use of ABPM and HBPM were important for overcoming barriers to the use of these assessment tools.

While this study provides useful local data on current diagnosis and management of hypertension and awareness of $\mathrm{BPV}$, there are a number of limitations that need to be taken into account when interpreting our results. The overall survey response rate was $40 \%$, meaning that the results are prone to nonresponder bias. Study data may not represent the total physician population in Singapore, because the subgroup that declined to participate may have responded differently to specific questions or sections of the survey. The study results are affected by limitations associated with self-report, including common method variance and the potential for physicians to interpret questions in different ways. We also rely on physician recall, which may not always be completely accurate (e.g., BP control rates) or reflect actual clinical practice or outcomes.

\section{Conclusion}

Physician diagnosis and management of hypertension in Singapore often varied from current international guideline recommendations. In addition, there was a gap in knowledge about BPV and its contribution to mortality. Physicians also acknowledged deficits in their understanding and management of BPV, accepting the need for training.

\section{Acknowledgments}

The authors would like to thank the management and staff of Kantar Health, Singapore, for their efforts in screening the respondents and carrying out interviews with the eligible respondents. This study was funded by Pfizer, Singapore. Editorial and medical writing support was provided by Nicola Ryan, independent medical writer, funded by Pfizer.

\section{Author contributions}

Dr Sajita Setia and Dr Kannan Subramaniam were involved in conception, design, analysis and interpretation of data. Professor Boon Wee Teo and Professor Jam Chin Tay were involved in analysis and interpretation of data. All authors were involved in the preparation of the manuscript, revising it for important intellectual content and final approval before submitting for publication.

\section{Disclosure}

Dr Sajita Setia and Dr Kannan Subramaniam are employees of Pfizer. Professor Jam Chin Tay has received advisory board and consultant honoraria from Pfizer. The authors report no other conflicts of interest in this work.

\section{References}

1. World Health Organization [webpage on the Internet]. Global Health Observatory (GHO) Data. Raised Blood Pressure. Available from: http:// www.who.int/gho/ncd/risk_factors/blood_pressure_prevalence_text/ en/. Accessed February 20, 2017.

2. World Health Organization. A Global Brief on Hypertension. Silent Killer, Global Public Health Crisis; 2013. Available from: http://ishworld.com/downloads/pdf/global_brief_hypertension.pdf. Accessed February 28, 2017. 
3. Health Hub Singapore [webpage on the Internet]. High Blood Pressure. Available from: http://www.healthhub.sg/a-z/diseases-andconditions/53/highbloodpressure. Accessed February 20, 2017.

4. Chobanian AV, Bakris GL, Black HR, et al. The seventh report of the joint national committee on prevention, detection, evaluation, and treatment of high blood pressure: the JNC 7 report. JAMA. 2003;289(19):2560-2572.

5. Neal B, MacMahon S, Chapman N. Blood pressure lowering treatment trialists C. Effects of ACE inhibitors, calcium antagonists, and other blood-pressure-lowering drugs: results of prospectively designed overviews of randomised trials. Blood Pressure Lowering Treatment Trialists' Collaboration. Lancet. 2000;356:1955-1964.

6. Psaty BM, Smith NL, Siscovick DS, et al. Health outcomes associated with antihypertensive therapies used as first-line agents. A systematic review and meta-analysis. JAMA. 1997;277(9):739-745.

7. James PA, Oparil S, Carter BL, et al. 2014 evidence-based guideline for the management of high blood pressure in adults: report from the panel members appointed to the Eighth Joint National Committee (JNC 8). JAMA. 2014;311(5):507-520.

8. Mancia G, Fagard R, Narkiewicz K, et al. 2013 ESH/ESC guidelines for the management of arterial hypertension: the Task Force for the Management of Arterial Hypertension of the European Society of Hypertension (ESH) and of the European Society of Cardiology (ESC). Eur Heart J. 2013;34(28):2159-2219.

9. National Institute for Health and Clinical Excellence [webpage on the Internet]. Hypertension: Clinical Management of Primary Hypertension in Adults (Update). Clinical Guideline 127; 2011. Available from: https://www.nice.org.uk/guidance/cg127/chapter/1-guidance. Accessed December 2016.

10. Shimamoto K, Ando K, Fujita T, et al; Japanese Society of Hypertension Committee for Guidelines for the Management of Hypertension. The Japanese Society of Hypertension guidelines for the management of hypertension (JSH 2014). Hypertens Res. 2014;37(4):253-390.

11. UK Prospective Diabetes Study (UKPDS). Intensive blood-glucose control with sulphonylureas or insulin compared with conventional treatment and risk of complications in patients with type 2 diabetes (UKPDS 33). UK Prospective Diabetes Study (UKPDS) Group. Lancet. 1998;352: $837-853$.

12. UK Prospective Diabetes Study (UKPDS). Effect of intensive bloodglucose control with metformin on complications in overweight patients with type 2 diabetes (UKPDS 34). UK Prospective Diabetes Study (UKPDS) Group. Lancet. 1998;352(9131):854-865.

13. UK Prospective Diabetes Study (UKPDS). Tight blood pressure control and risk of macrovascular and microvascular complications in type 2 diabetes: UKPDS 38. UK Prospective Diabetes Study Group. BMJ. 1998;317(7160):703-713.

14. UK Prospective Diabetes Study (UKPDS). Efficacy of atenolol and captopril in reducing risk of macrovascular and microvascular complications in type 2 diabetes: UKPDS 39. UK Prospective Diabetes Study Group. BMJ. 1998;317(7160):713-720.

15. Lindholm LH, Carlberg B. The new Japanese Society of Hypertension guidelines for the management of hypertension (JSH 2014): a giant undertaking. Hypertens Res. 2014;37:391-392.

16. Bakris GL. The importance of blood pressure control in the patient with diabetes. Am J Med. 2004;116(suppl 5A):30S-38S.

17. Ravera M, Re M, Deferrari L, Vettoretti S, Deferrari G. Importance of blood pressure control in chronic kidney disease. J Am Soc Nephrol. 2006;17(4 suppl 2):S98-S103.

18. Fraser SD, Roderick PJ, McIntyre NJ, et al. Suboptimal blood pressure control in chronic kidney disease stage 3 : baseline data from a cohort study in primary care. BMC Fam Pract. 2013;14:88.

19. Ong KL, Cheung BM, Man YB, Lau CP, Lam KS. Prevalence, awareness, treatment, and control of hypertension among United States adults 1999-2004. Hypertension. 2007;49(1):69-75.

20. Maahs DM, Kinney GL, Wadwa P, et al. Hypertension prevalence, awareness, treatment, and control in an adult type 1 diabetes population and a comparable general population. Diabetes Care. 2005;28(2):301-306.
21. Teo BW, Chua HR, Wong WK, et al. Blood pressure and antihypertensive medication profile in a multiethnic Asian population of stable chronic kidney disease patients. Singapore Med J. 2016;57(5):267-273.

22. Malhotra R, Chan A, Malhotra C, Ostbye T. Prevalence, awareness, treatment and control of hypertension in the elderly population of Singapore. Hypertens Res. 2010;33(12):1223-1231.

23. Frattola A, Parati G, Cuspidi C, Albini F, Mancia G. Prognostic value of 24-hour blood pressure variability. J Hypertens. 1993;11(10):1133-1137.

24. Grove JS, Reed DM, Yano K, Hwang LJ. Variability in systolic blood pressure-arisk factor for coronary heart disease? AmJ Epidemiol. 1997;145(9): 771-776.

25. Stevens SL, Wood S, Koshiaris C, et al. Blood pressure variability and cardiovascular disease: systematic review and meta-analysis. $B M J$. 2016;354:i4098.

26. Chia YC, Lim HM, Ching SM. Long-term visit-to-visit blood pressure variability and renal function decline in patients with hypertension over 15 years. $J$ Am Heart Assoc. 2016;5(11):e003825.

27. Hsieh YT, Tu ST, Cho TJ, Chang SJ, Chen JF, Hsieh MC. Visit-to-visit variability in blood pressure strongly predicts all-cause mortality in patients with type 2 diabetes: a 5.5 -year prospective analysis. Eur $J$ Clin Invest. 2012;42(3):245-253.

28. Hastie CE, Jeemon P, Coleman H, et al. Long-term and ultra long-term blood pressure variability during follow-up and mortality in 14,522 patients with hypertension. Hypertension. 2013;62(4):698-705.

29. Gan SK, Loh CY, Seet B. Hypertension in young adults - an underestimated problem. Singapore Med J. 2003;44(9):448-452.

30. Singapore Ministry of Health. Biomedical Research Regulation. Operational Guidelines for Institutional Review Boards; 2007. Available from: https://www.moh.gov.sg/content/dam/moh_web/Publications/Guidelines/Human Biomedical Research/2007/IRB Operational Guidelines_14-12-07_formatted.pdf. Accessed May 26, 2017.

31. Ardery G, Carter BL, Milchak JL, et al. Explicit and implicit evaluation of physician adherence to hypertension guidelines. J Clin Hypertens (Greenwich). 2007;9(2):113-119.

32. Singapore Ministry of Health. Ministry of Health (MOH) Clinical Practice Guidelines 2/2005: Hypertension (Including Withdrawal Notice). Available from: https://www.moh.gov.sg/content/dam/moh_web/HPP/ Doctors/cpg_medical/withdrawn/cpg_Hypertension-Jun 2005.pdf. Accessed February 20, 2017.

33. Wright JT Jr, Williamson JD, Whelton PK, et al. A randomized trial of intensive versus standard blood-pressure control. $N$ Engl J Med. 2015;373(22):2103-2116.

34. Accord Study Group, Cushman WC, Evans GW, et al. Effects of intensive blood-pressure control in type 2 diabetes mellitus. $N$ Engl J Med. 2010;362(17):1575-1585.

35. Bhatt H, Ghazi L, Calhoun D, Oparil S. BP targets in hypertension: what should we do now that SPRINT is out? Curr Cardiol Rep. 2016;18(10):98.

36. Zanchetti A, Liu L, Mancia G, et al. Blood pressure and LDL-cholesterol targets for prevention of recurrent strokes and cognitive decline in the hypertensive patient: design of the European Society of HypertensionChinese Hypertension League Stroke in Hypertension Optimal Treatment randomized trial. J Hypertens. 2014;32(9):1888-1897.

37. Myers MG. A short history of automated office blood pressure - 15 years to SPRINT. J Clin Hypertens (Greenwich). 2016;18(8):721-724.

38. Myers MG, Campbell NR. Unfounded concerns about the use of automated office blood pressure measurement in SPRINT. J Am Soc Hypertens. 2016;10(12):903-905.

39. Kario K. Evidence and perspectives on the 24-hour management of hypertension: hemodynamic biomarker-initiated 'anticipation medicine' for zero cardiovascular event. Prog Cardiovasc Dis. 2016;59(3): 262-281.

40. Lawes CM, Bennett DA, Parag V, et al. Blood pressure indices and cardiovascular disease in the Asia Pacific region: a pooled analysis. Hypertension. 2003;42(1):69-75.

41. Sisson SD, Rastegar D, Rice TN, Prokopowicz G, Hughes MT. Physician familiarity with diagnosis and management of hypertension according to JNC 7 guidelines. J Clin Hypertens (Greenwich). 2006;8(5):344-350. 


\section{Publish your work in this journal}

Vascular Health and Risk Management is an international, peerreviewed journal of therapeutics and risk management, focusing on concise rapid reporting of clinical studies on the processes involved in the maintenance of vascular health; the monitoring, prevention and treatment of vascular disease and its sequelae; and the involvement of metabolic disorders, particularly diabetes. This journal is indexed on PubMed Central and MedLine. The manuscript management system is completely online and includes a very quick and fair peer-review system, which is all easy to use. Visit http://www.dovepress.com/ testimonials.php to read real quotes from published authors.

Submit your manuscript here: https://www.dovepress.com/vascular-health-and-risk-management-journal 